\title{
Research on the Construction of Virtual Reality Simulation System for Psychological Stress Training
}

\author{
Chen Yong-ke ${ }^{1}$, Rui Jie ${ }^{2}$, Qiao Bin ${ }^{2}$ \\ ${ }^{1} 1$ st Department, Army Officer Academy, Hefei, China \\ ${ }^{2}$ Postgraduate Student Regiment, Army Officer Academy, Hefei, China
}

Email address:

54998009@qq.com (Chen Yong-ke), 93922432@qq.com (Rui Jie)

To cite this article:

Chen Yong-ke, Rui Jie, Qiao Bin. Research on the Construction of Virtual Reality Simulation System for Psychological Stress Training. Psychology and Behavioral Sciences. Vol. 6, No. 2, 2017, pp. 30-35. doi: 10.11648/j.pbs.20170602.13

Received: March 17, 2017; Accepted: May 3, 2017; Published: May 10, 2017

\begin{abstract}
Due to the lack of realistic training environment in psychological stress training problems, virtual reality (VR) technology is introduced into the psychological stress training. This paper summarizes the five psychological stress training courses for VR technology, carries on the demand analysis, structure design and function module design of the virtual reality simulation system for psychological stress training (VRSSPST), researches on the training organization, and enriches the psychological training method.
\end{abstract}

Keywords: Virtual Reality Technology, Psychological Stress Training, VRSSPST, System Construction

\section{Introduction}

With the acceleration of the pace of life and work pressure, peoples' psychological stress quality and ability are more and more concerned by scholars both at home and abroad $[1,2]$. Psychological stress contains three different meanings: first, stress is stressful event or an environmental stimulus. This is regarded as the stress of the body in the harmful environment of stimulation, it can be said that the scene changes of the internal and external environment. Second, stress refers to a subjective reflection. It is a kind of internal psychological state of psychological tension or arousal, and it is an interpretative and defensive response process in the human body. Third, the non-specific response of the body to any of the requirements imposed. Combined three layer of meaning, psychological stress is human being stimulated by the unexpectedly tense situation and stress stimulation which plays an important role in the social life scenes and serious life events, and because of the imbalance between the object requirements and the ability to deal with the problem, human produce a kind of adaptive response.

The introduction of virtual reality technology in psychological stress training [3-6], realistic training environment creating, realistic training courses setting, is an effective way to improve the training effect [7-9], and has very important practical significance to carry out the research of psychological stress training based on virtual reality technology.

\section{Courses Requirements in Psychological Stress Training Based on VR Technology}

\subsection{The Definition of Psychological Stress Training and the System}

In a broad sense, all can improve the ability of people to withstand the pressure of the outside world, reduce psychological stress adverse reactions are psychological stress training [10-12]. There are three functions of psychological stress training, to improve the level of mental activity, to cultivate good psychological quality and to overcome the psychological barrier.

In this paper, the virtual reality simulation system for psychological stress training (VRSSPST) refers to a training simulation system of virtual reality technology based, training under the realistic environment, scientific use of psychology, the theory of education and skills training, purpose and plan of the psychological stimulus and influence, improve the level of people's psychological stress. It follows 
four basic principles, the combination of gradualness and persistence, the combination of universality and particularity, the combination of fidelity and security, and the combination of proactive and conscious cooperation.

\subsection{Suitable for Psychological Stress Training Courses Based on Virtual Reality Technology}

VR technology is "to use the computer technology as the core of modern high technology, the generation of realistic visual, auditory, tactile and other integrated virtual environment. Users with the necessary equipment in a natural way to interact with the virtual world objects, resulting in personal experience and experience in the real environment." VR technology can be used to generate multidimensional dynamic space environment, so that trainees and the "objective object" interaction, resulting in a sense of immersive. The application of VR technology in the technological stress training can guarantee the scientific nature of the training, increase the pertinence of training, enrich the training content, reduce the training cost, and facilitate the training evaluation. According to the psychological stress training principle and method, put forward five applies in psychological stress training courses based on VR, as shown in Table 1.

Table 1. The psychological stress training courses based on VR.

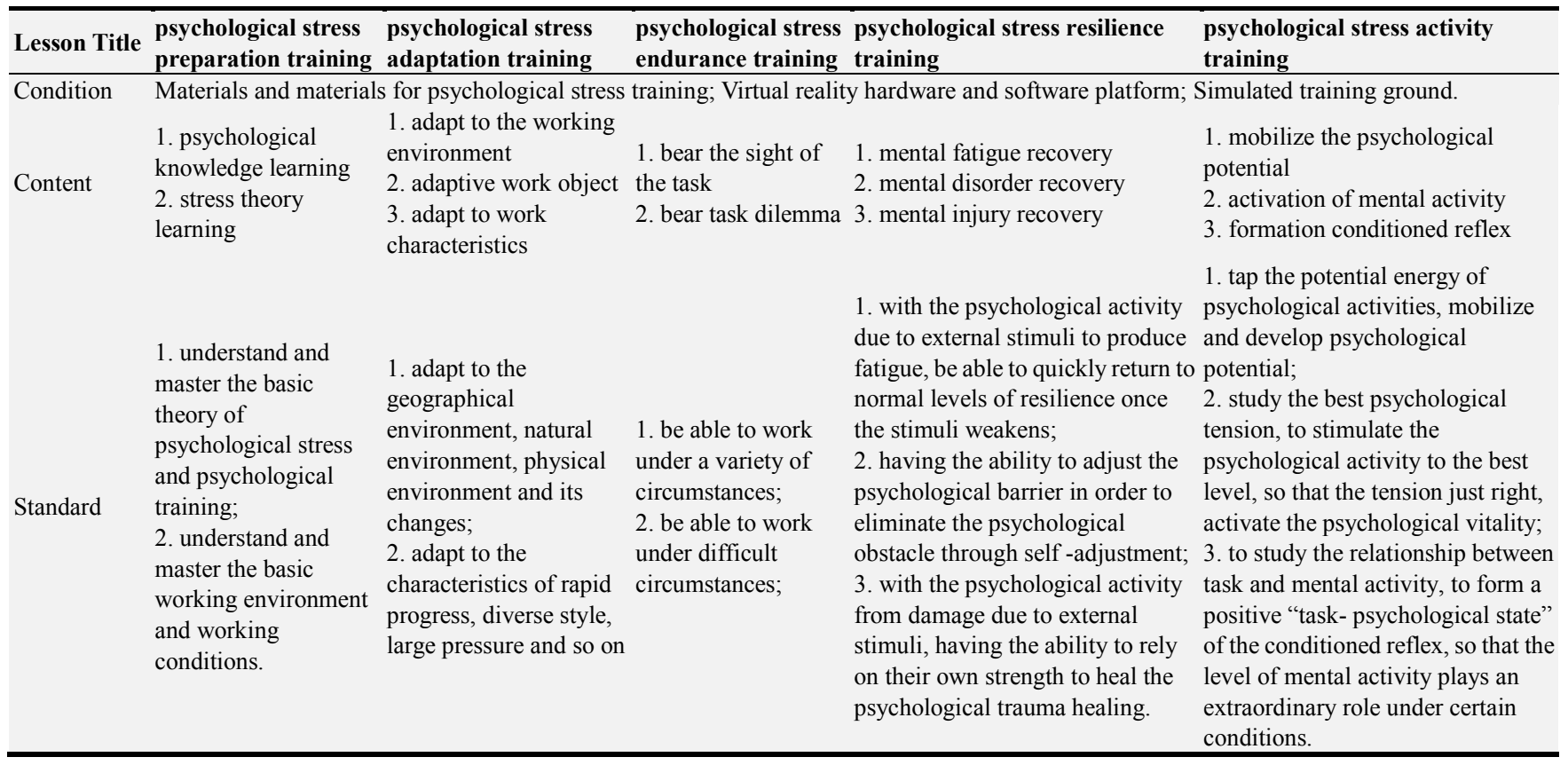

\section{The System Design of VRSSPST}

\subsection{Requirement Analysis}

(1) System functional requirements

The function of the system demand analysis should be based on fully understand and grasp the basic requirements of the training courses and contents. According to the different implementations, the five training courses can be divided into two parts of education and training, besides, according to the raining stages, them also covers the two levels of individual training and collective training. In addition, because the basic information of trainees and the data generated in the training process is very large, it also requires the system to implement effective management. Therefore, VRSSPST should have four basic functions: theoretical education, virtual training, measurement and evaluation and information management. At the same time, we should distinguish between the individual psychological stress training and the collective psychological stress training in two levels.
(2) System structure requirements

VRSSPST is composed of three parts: software system, hardware system and database system. The software system includes virtual environment modeling software, environment generation and processing software, 3D imagegeneration software, observation and control software and data analysis software; the hardware system includes computer, operating handle, mouse, data glove, projection equipment, curtain, helmets, glasses, heart rate bracelet, skin conductance sensor, muscle electric sensor, electroencephalogram headband, pulse sensor etc. Database system mainly includes user database, theory education database, virtual training database and training record database.

(3) System performance requirements

The training system should have excellent fidelity, real-time and expansibility. Fidelity including realistic training environment, realistic training tasks and realistic psychological feedback, strive to provide immersive sensory experience for the trainees. Real-time including real-time system, real-time response and real-time system configuration, which determines the quality of human-computer interaction; scalability, including the 
expansion of the architecture and the expansion of the virtual scale, can greatly shorten the system development cycle and the use efficiency.

\subsection{Structure Design}

VRSSPST consists of two parts: hardware and software.
Among them, the hardware includes high-performance computers, input devices and output devices. The software includes the operating system installed in the computer, database management system, application software, as shown in Figure 1.

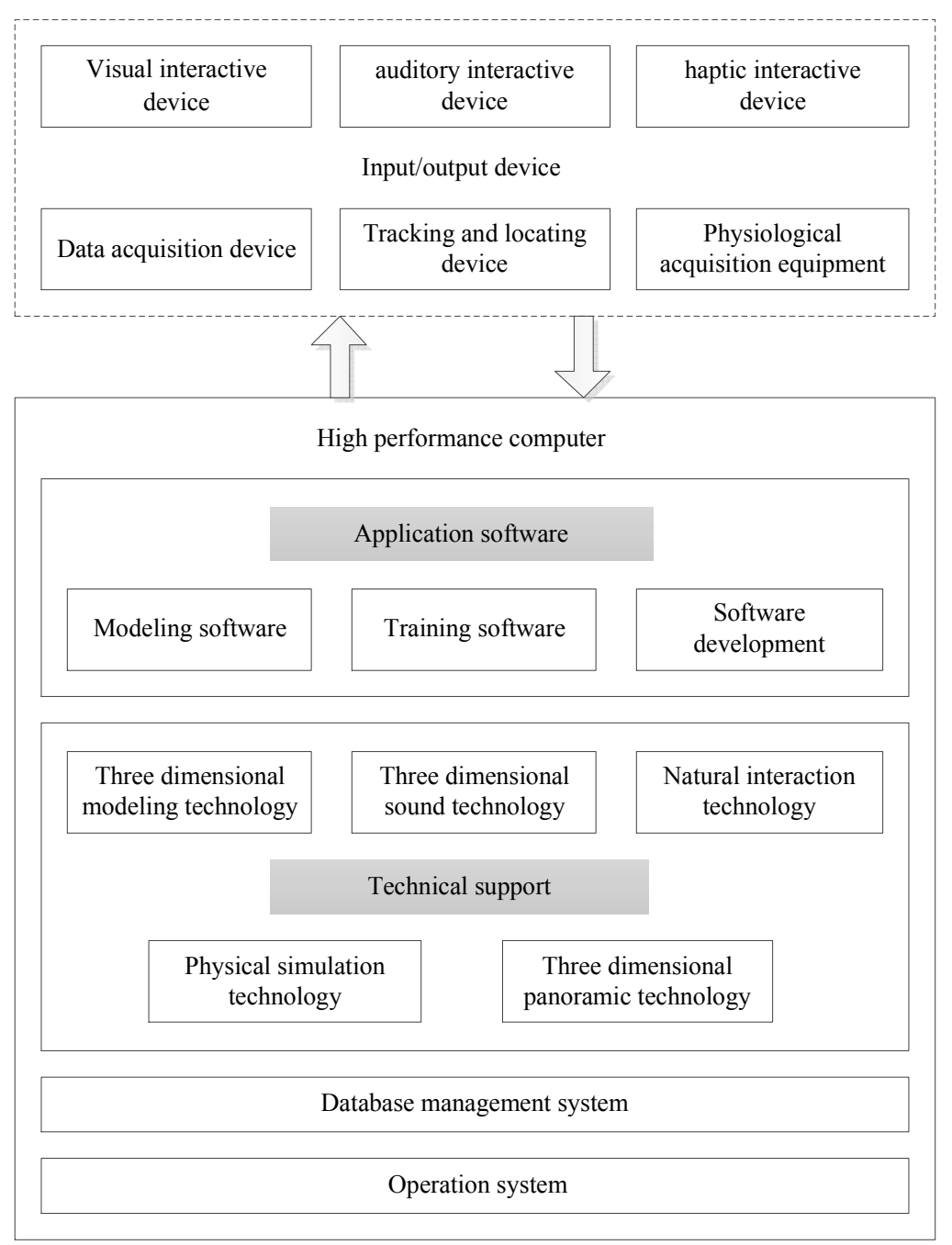

Figure 1. The structure of VRSSPST.

High performance computer is running a carrier of the whole system, it should cooperate with other hardware and software technology to realize the training function and generate a virtual training environment; The application software is mainly responsible for the connection and integration of VR system components, directly affect the system real-time and nature. The input device is the operation system of the system to receive information from the user. The trainee completes the interactive task of virtual training by using the input device. The interactive information is transmitted to the computer through the data acquisition device and the motion tracking device, and generates real-time feedback information. The physiological acquisition device can accurately collect the physiological information of the electrocardiogram, heart rate, heart rate variability, skin conductance, brain waves, muscle power, pulse, etc., and transmit them to the computer. An output device is a system display device that is used to convey information to the user. The virtual training environment and virtual training interface generated by computer are presented in front of the trainees through visual, auditory, tactile and other interactive devices.

\subsection{Function Module Design}

According to the demand structure, VRSSPST is divided into four subsystems, namely individual psychological stress training subsystem, collective psychological stress training subsystem, acquisition and analysis evaluation subsystem and training information management subsystem, each subsystem contains several functional modules, as shown in Figure 2.

(1) Individual psychological stress training subsystem

The task of individual psychological stress training 
subsystem is to help trainees to complete the individual psychological stress training content, which is divided into two modules, theoretical education module and virtual training module. The main function of the theory education module is to complete the contents of psychological stress and psychological knowledge education by means of voice explanation, multimedia courseware and video demonstration. The main function of virtual training module is to create a single training environment, training tasks and training difficulties, under the simulation of virtual training environment, the trainees ultimately achieve the purpose of improving the ability of psychological stress.

(2) Collective psychological stress training subsystem

The object of collective psychological stress training subsystem is the team; the function module is basically the same as individual psychological stress training system. This subsystem also including two parts: theory education module and virtual training module. The main function of the theory education module is to introduce the training task to help the trainees to complete the collective psychological stress training. The main function of virtual training module is to generate the collective training environment, training tasks and training dilemma. Under the strong stimulation of the extremely complex and brutal virtual training environment, the trainees can improve the collective ability of psychological stress.

(3) Acquisition and analysis evaluation subsystem

There are two parts in the acquisition and analysis evaluation subsystem, one is the acquisition of physiological indexes, and the other is the analysis evaluation of training effect. By connecting the heart rate bracelet, skin conductance sensor, muscle electric sensor, electroencephalogram headband, pulse sensor and other physiological acquisition equipment, heart rate, brain waves, muscle power and skin conductance can be accurately measured. Then the data is stored in the system database. Finally, the system is used to evaluate the training effect in five aspects.

(4) Training information management subsystem

Training information management subsystem is composed of three parts: user information management module, training resource management module and training record management module. The main function of the user information management module is to query and update the basic information of user number, the trainees' user name, category, name, birth date, photos, gender, professional, unit and contact. The function of the training resource management module is to manage the training environment model, the training task model and the training dilemma model effectively, so as to call and combine at any time. The training record management module is mainly used to manage the multimedia materials such as picture, text, audio, video and so on.

\section{The Application of VRSSPST}

\subsection{Training Implementation Process}

The training implementation process of the VRSSPST can be divided into two parts: training preparation and training implementation. The training preparation includes the determination of the research object, the formulation of the training plan and the configuration training system. The configuration training system is the precondition and guarantee of application. Hardware preparation is mainly to set up visual, auditory, tactile and other interaction devices, tracking and acquisition equipment as well as physiological acquisition equipment in place in accordance with the training needs. It is connected with the high performance computer through the network communication equipment, to construct the hardware architecture of the whole training system. Software preparation is mainly install the operation system software, database management system, software development software, network software, multimedia software, 3D software and other auxiliary software in the hardware according to the overall design of the technical requirements. System operation process includes user registration and user use two links, as shown in Figure 3.

\subsection{Steps in the Implementation Process of Training}

(1) Organization trainer leading to complete training preparation

Firstly, the organization trainer count the number of articles, prepare training software system and hardware equipment. In addition the organization trainer grasp the trainee's physical and mental condition by the way of observation, question and test, and eliminate the persons who are unsuitable to participate in training; Secondly, the organization trainer announce the training courses, training contents and training objective, and definite the training classification; thirdly, the organization trainer propose to pay attention to the training of the items, system operation regulations and safety; Finally, the organization trainer guide the trainees into a predetermined position, wear articles, prepare training, organize security personnel in place. At this stage, the organization trainer should play a leading role in training the trainees to complete the training preparation.

(2) Trainees to complete the training content by self

The trainee should follow "the first theoretical education, after virtual training" and "the first individual training, after collective training", "the first crew training, after leader training" sequence, in order to complete five training courses and content. At this stage, the trainee is the absolute subject of the training practice, through the self-learning, self-training, personally experience of various factors and unexpected situations. To ensure training completed successfully, organization trainers mainly play the role of observation and guidance, to intervention and guidance depending on the situation.

(3) Training evaluation

The training assessment shall be made by the organization trainer and trainees work jointly. The trainee will occur in the training situation and problems, and the real psychological feelings and experiences with other participants and group training together to exchange and share; According to the 
training information and training evaluation effect, organization trainer shall outspoken pointe out problems and deficiencies of trainees.

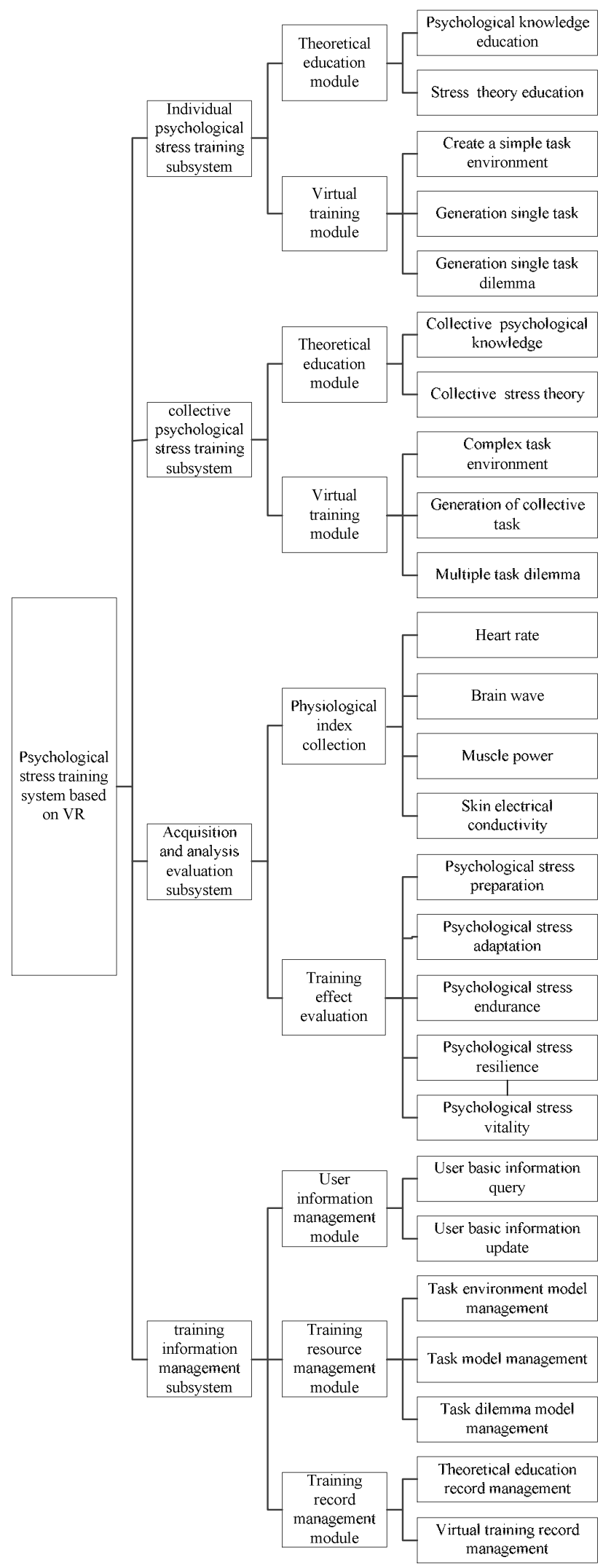

Figure 2. The function module of VRSSPST.

\section{Conclusion}

Based on the study of VR technology and the psychological stress training theory, the paper summarizes the five psychological stress training courses based on VR, puts forward the concept of the VRSSPST, and carries on the demand analysis, structure design, functional module design. This paper studies the application of VRSSPST, standardizes the system operation flow and the organization and implementation procedure, expandes the application field of VR technology, and enriches the psychological stress training methods. The follow-up will continue to focus on the development of VR technology, in-depth study and analysis of the actual needs of training, targeted to expand the system function and improve system effectiveness and immersion.

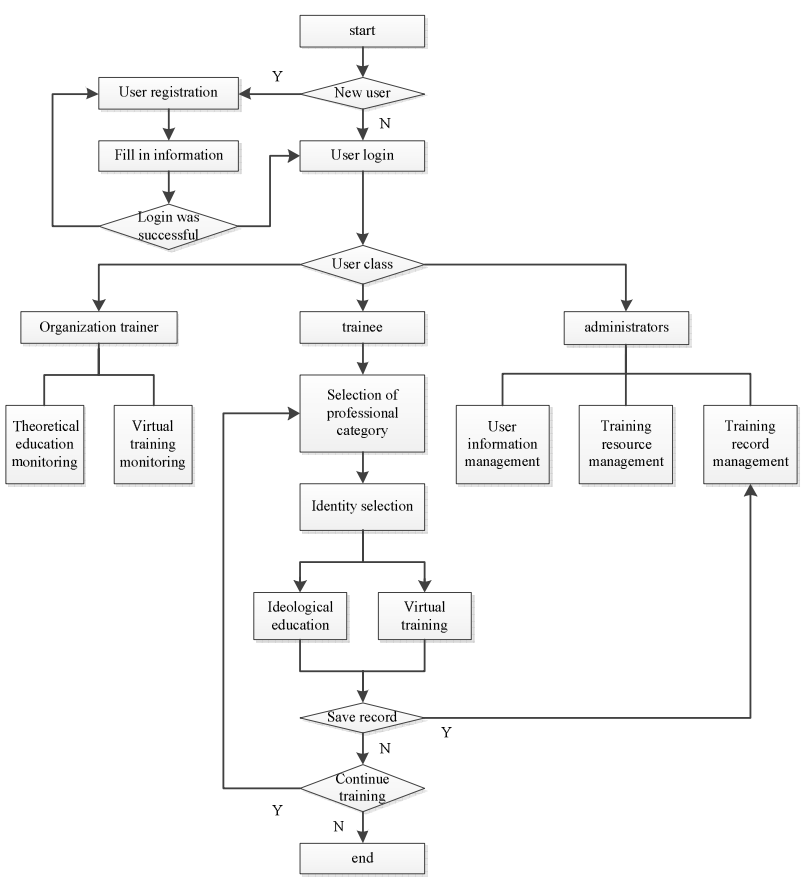

Figure 3. The application flow of the VRSSPST.

\section{References}

[1] Tan Hong, Research on Psychological Stress and its Correlative Factors among Civil Aviation Pilots in Mainland China, Tianjin: Tianjin Normal University, 2007, pp. 13-14.

[2] Jiang Qiaoling, Chen Fengmei and Li Zhilin, analysis of scald of college students mental stress to life events in a health professional college of Suzhou, 1st ed., vol. 29. Hubei: medicine and society, 2016, pp. 62-64.

[3] Liu Jianxin and Liu Wangsheng, Application of virtual reality technology in military education and training, Changchun: Jilin People's Press, 2006, pp.232-234.

[4] Sun Tieqiang and Gu Baiyuan, The application of virtual reality technology in the evaluation of the psychological quality of armored solders, 3rd ed., Journal of Armored Force Technical College, 2002, pp.26-27. 
[5] Peng Geng and Zhang Limin, Application of virtual reality technology in psychological quality education, 6rd ed., vol. 14. Education of Naval Colleges, 2004, pp.42-44.

[6] Wang Jun, Wan Jing, Xue Xia, et al, The development of psychological stress training system for pilots, 2rd ed., vol. 60 . Beijing: people's military surgeon, 2017, pp. 204-206.

[7] Wang Tao and Li Min, Application of virtual reality technology in the prevention and treatment of disorders in the US army, 5rd ed., vol. 31. Journal of Preventive Medicine of Chinese People's Liberation Army, 2013, pp.473-475.

[8] GERARDI M, ROTHBAUM B O, ESSLER K, et al, Virtual reality exposure therapy using a virtual Iraq case report, 2rd ed., vol. 21. J Traumatic Stress, 2008, pp. 209.

[9] RIZZO A S, DIFEDE J, ROTHBAUM B O, et al,
Development and early evaluation of the Virtual Iraq/Afghanistan exposure therapy system for combat-related PTSD, Ann N Y Acad Sci, 2010, pp. 114.

[10] Gao Yanmei, Study on the Correlation between Coping Efficacy, Coping Style and Mind and Body in Stress of Master's Graduate Students, Wuhan: Huazhong University of Science and Technology, 2007, pp. 3-4.

[11] Liu Xiaolin. The Structure of military psychological stress and the Correlative Study. Guangzhou: Guangzhou University, 2008, pp. 1-2.

[12] Huang Wenying, Changes of Immune During Physical Exercise and Psychological Stress and Research of Their Mechanism, Shanghai: East China Normal University, 2005, pp. 4-5. 